\title{
A Characterization of the Efficiency-Fairness Tradeoff
}

\author{
Dimitris Bertsimas* Vivek F. Farias ${ }^{\dagger} \quad$ Nikolaos Trichakis $^{\ddagger}$ \\ July 9,2010
}

\begin{abstract}
A central decision maker allocates resources to each of $n$ players who derive utility from their allocation. Consequently, her actions may be viewed as choosing an allocation of utilities $u=\left(u_{1}, u_{2}, \ldots, u_{n}\right)$ from some set of feasible allocations $U \in \mathbf{R}_{+}^{n}$. Consider a decision maker that chooses an allocation $u \in U$ to maximize

$$
\sum_{j=1}^{n} \frac{u_{j}^{1-\alpha}}{1-\alpha} .
$$

The above family of objective functions proposed originally by Atkinson and parameterized by $\alpha \in \mathbf{R}_{+}$, permits the decision maker to tradeoff efficiency (by lowering $\alpha$ ) for fairness (by increasing $\alpha$ ). The family of Atkinson utility functions is canonical in that it captures the efficient or "utilitarian" allocation $(\alpha=0)$, the "max-min" fair allocation $(\alpha \rightarrow \infty)$, and the proprotionally fair (or Nash bargaining) allocation $(\alpha \rightarrow 1)$.

This paper characterizes the tradeoff between efficiency and fairness in this general setting. In particular, we demonstrate that under reasonable assumptions on $U$, the total utility to players under a fair allocation, $\operatorname{FAIR}(U, \alpha)$, and the total utility to players under a perfectly efficient allocation, $\operatorname{FAIR}(U, 0) \triangleq \operatorname{SYSTEM}(U)$, must satisfy

$$
\frac{\operatorname{SYSTEM}(U)-\operatorname{FAIR}(U, \alpha)}{\operatorname{SYSTEM}(U)} \leq 1-\Theta\left(n^{-\frac{\alpha}{\alpha+1}}\right)
$$
\end{abstract}

and moreover, that this bound is essentially tight.

\section{Introduction}

Consider the following resource allocation problem: Due to unexpected weather, we must re-allocate landing and take-off slots to airlines operating in some region. The change in available capacity that calls for this re-allocation naturally leads to airline delays, both on

\footnotetext{
*Sloan School and Operations Research Center, Massachusetts Institute of Technology, dbertsim@mit.edu ${ }^{\dagger}$ Sloan School and Operations Research Center, Massachusetts Institute of Technology, vivekf@mit.edu

${ }^{\ddagger}$ Operations Research Center, Massachusetts Institute of Technology, nitric@mit.edu; research partially supported by NSF grants DMI-0556106, EFRI-0735905.
} 
the ground and in the air. Since airline delays translate to well documented dollar figures per passenger-hour, it is tempting to consider accomplishing the said re-allocation in a manner that minimizes total dollar costs. While this objective is natural, a somewhat surprising fact is that existing practice (at least within the United States) does not take into account delay costs in making such re-allocation decisions. The emphasis rather, is on an allocation that may be viewed as equitable or fair to the airlines concerned. This natural dichotomy, between "efficiency" of the one hand and "fairness" on the other is a staple of many real world resource allocation problems including the one above. How one chooses to address this dichotomy can have far reaching policy impacts. This state of affairs begs for a broad understanding of the inherent tradeoff between efficiency on the one hand and fairness on the other; an understanding of such a tradeoff would, in principle, inform policy decisions such as the one above. Our goal here is to provide a mathematical foundation for characterizing this tradeoff.

Consider a resource allocation problem, in which a central decision maker (CDM) needs to decide on the allocation of scarce resources among $n$ players. Each player derives a nonnegative utility, depending on the allocation decided by the CDM (e.g., via means of a utility function). For a given allocation of resources, there is thus a corresponding utility allocation $u \in \mathbf{R}_{+}^{n}$, with $u_{j}$ equal to the utility derived by the $j$ th player, $j=1, \ldots, n$.

A utility allocation $u \in \mathbf{R}_{+}^{n}$ is feasible if and only if there exists an allocation of resources for which the utilities derived by the players are $u_{1}, u_{2}, \ldots, u_{n}$ accordingly. We define the utility set $U \subset \mathbf{R}_{+}^{n}$ as the set of all feasible utility allocations. Encapsulated in the notion of the utility set are the preferences of the players and the way they derive utility, as well as individual constraints of the players or the CDM, constraints on the resources, etc. Thus, the utility set provides a condensed way of describing the problem. Given the utility set, the CDM then needs to decide which utility allocation among the players to select, or, equivalently which point from the utility set to select.

The above setup has been studied within the research areas of fair bargaining and welfare economics (see [13], [19]). Numerous applications can also be cast under the above general framework, for example bandwidth allocation problems [9], scheduling, facility location problems [10], etc. The most prominent way of addressing the described resource allocation problem has been the identification of a real-valued social welfare function of the utility allocation, which is used by the CDM to rank allocations. In the next section, we review social welfare functions that give rise to "efficient" and "fair" allocations.

\section{Efficiency-maximizing and fair allocations}

A natural objective for the central decision maker is to maximize an efficiency metric of the system (defined appropriately). On the other hand, in the case of a competitive environment, where self-interested players are involved, such practice might result in great inequalities among the utilities derived by different players. Then, the incorporation of fairness considerations in the allocation decision would be highly desirable. The introduction of fairness, however, will most likely have a negative impact on the efficiency of the system. Indeed, the efficiency-fairness tradeoff is a central issue of resource allocation problems [8]. We next discuss efficiency-maximizing and fair allocations. 
Efficiency-maximizing allocation. In this work, we adopt the sum of utilities (derived by the players) as the system efficiency metric. Although in some cases idiosyncratic characteristics of a problem might suggest otherwise, the sum of utilities is commonly a natural way of quantifying efficiency. The sum of utilities is also among the most well studied social welfare fucntions, known as the (Bentham) utilitarian function.

Given a utility set $U$, an allocation of utilities that maximizes efficiency (also known as utilitarian) then corresponds to an optimal solution of the problem

$$
\begin{array}{ll}
\operatorname{maximize} & \mathbf{1}^{T} u \\
\text { subject to } & u \in U,
\end{array}
$$

with $u \in \mathbf{R}_{+}^{n}$ and $\mathbf{1}$ is the vector of all ones. We denote the optimal value of this problem with $\operatorname{SYSTEM}(U)$, i.e.,

$$
\operatorname{SYSTEM}(U)=\sup \left\{\mathbf{1}^{T} u \mid u \in U\right\}
$$

which corresponds to the highest possible system efficiency.

The utilitarian principle of maximizing sum of utilities, however, is neutral towards inequalities among the utilities derived by the players. As a result, it is considered to lack fairness considerations [19].

Fair allocation. Due to the subjective nature of fairness and different possible interpretations of equity, there is no principle that is universally accepted as "the most fair". In particular, there has been a plethora of proposals in the literature under axiomatic bargaining, welfare economics, as well as in applications ranging from networks, air traffic management, healthcare and finance. We refer the reader to [19] and [4] for a more detailed exposition.

A fairness scheme of particular interest, and one on which we will focus our attention in this work, is the $\alpha$-fairness scheme, which was introduced in the work by Atkinson [1] (see also [13] and [2] for more details). According to $\alpha$-fairness, the CDM decides on the allocation by maximizing the constant elasticity social welfare function $W_{\alpha}$, parameterized by $\alpha \geq 0$, and defined for $u \in \mathbf{R}_{+}^{n}$ as

$$
W_{\alpha}(u)= \begin{cases}\sum_{j=1}^{n} \frac{u_{j}^{1-\alpha}}{1-\alpha}, & \text { for } \alpha \geq 0, \alpha \neq 1, \\ \sum_{j=1}^{n} \log \left(u_{j}\right), & \text { for } \alpha=1 .\end{cases}
$$

A resulting utility allocation, denoted by $z(\alpha)$, is such that

$$
z(\alpha) \in \underset{u \in U}{\operatorname{argmax}} W_{\alpha}(u),
$$

and is referred to as an $\alpha$-fair allocation.

Under the constant elasticity welfare function, the proportional increase in welfare attributed to a given player for a given proportional increase of her utility, is the same at all utility levels. Moreover, since the constant elasticity function is concave and component-wise increasing, it exhibits diminishing marginal welfare increase as utilities increase. The rate 
at which marginal increases diminish is controlled by the parameter $\alpha$, which is called the inequality aversion parameter for that reason.

The $\alpha$-fairness scheme can be useful in practice for a CDM, as it facilitates the efficiencyfairness tradeoff. In particular, a higher value of the inequality aversion parameter is thought to correspond to a "fairer" scheme (see [18], [2], [12]). Note that for the smallest value of $\alpha=0$, we recover the utilitarian principle, which is neutral towards inequalities. Thus, the CDM can adjust attitudes towards inequalities by means of a single parameter.

Furthermore, the $\alpha$-fairness scheme captures as special cases two important fairness schemes, which have been studied extensively in the literature; for $\alpha=1$, the scheme corresponds to proportional fairness [16], [9], whereas for $\alpha \rightarrow \infty$, the $\alpha$-fair allocation converges to the utility allocation suggested by max-min fairness [7], [13].

Although the $\alpha$-fairness scheme has been studied both from a theoretical and a practical perspective, prominently in networks (see [15], [5], [11], [14], [17]), the underlying efficiencyfairness tradeoff is still not well understood. Recent work has been devoted to theoretically characterizing what it actually means for a higher value of $\alpha$ to be more fair [12]. The impact of a higher value of $\alpha$ on the system efficiency has also received a lot of attention. In [4] the authors present tight upper bounds on the efficiency loss for the special cases of proportional and max-min fairness. For the more interesting general case of varying $\alpha$ (and thus giving the CDM the flexibility of the efficiency-fairness tradeoff), however, only empirical studies are available that suggest that a higher value of $\alpha$ results in higher efficiency loss, although counterexamples exist (see [18] and references therein).

This paper sheds light on exactly this matter, by quantifying what the maximum efficiency loss can be for a fixed value of $\alpha$. We next formally define the notion of the efficiency loss, and present the main results in the following sections.

Efficiency loss. As the central decision maker incorporates fairness considerations, the efficiency of the system (measured as the sum of utilities), is likely to decrease, compared to the efficiency under the utilitarian solution.

Suppose the CDM adopts $\alpha$-fairness, using a fixed value for the inequality aversion parameter $\alpha$, and the utility set $U$ is such that an $\alpha$-fair allocation exists (e.g., $U$ is compact). Then, the efficiency of the system under the $\alpha$-fairness scheme will be the sum of the components of the $\alpha$-fair utility allocation $z(\alpha)$ (as in (1) ), and denoted by

$$
\operatorname{FAIR}(U ; \alpha)=\mathbf{1}^{T} z(\alpha) .
$$

The efficiency loss is the difference between the maximum system efficiency, SYSTEM $(U)$, and the efficiency under the fair scheme, $\operatorname{FAIR}(U ; \alpha)$. The efficiency loss relative to the maximum system efficiency is the so called price of fairness [4], defined in this case

$$
\operatorname{POF}(U ; \alpha)=\frac{\operatorname{SYSTEM}(U)-\operatorname{FAIR}(U ; \alpha)}{\operatorname{SYSTEM}(U)} .
$$

The price is a number between 0 and 1, and corresponds to the percentage efficiency loss compared to the maximum system efficiency. It is a key quantity to understanding the efficiency-fairness tradeoff. 


\section{Our contributions}

In this section, we summarize the main results of this paper: upper bounds on the price of $\alpha$-fairness, which are applicable under mild assumptions. An important quantity is the maximum achievable utility of each player, defined (for the $j$ th player) as

$$
u_{j}^{\star}=\sup \left\{u_{j} \mid u \in U\right\}, \text { for all } j=1, \ldots, n .
$$

The bounds depend on the inequality aversion parameter $\alpha$, the number of players involved, and their maximum achievable utilities (in case they are not equal). Section 4 includes the results with more technical details and proofs. Section 5 deals with near worst-case examples.

Consider a resource allocation problem, as described in Section 1, We make the following assumption:

Assumption 1. The utility set is compact and convex.

Assumption 1 is standard in the literature of fair bargains and also very frequently satisfied in practice. In particular, compactness of the utility set follows from limited resources and bounded and continuous functions that map resource allocations to utility for each player. Also, in case of nonconvex utility sets, randomization over possible utility allocations results in a convex set (of expected utilities). For an extended discussion of Assumption 1 we refer the reader to [19] and [4].

Under Assumption 1, and in case the maximum achievable utilities of the players are equal, we show that the price of $\alpha$-fairness is bounded,

$$
\operatorname{POF}(U ; \alpha) \leq 1-\Theta\left(n^{-\frac{\alpha}{\alpha+1}}\right),
$$

where $n$ is the number of players. In Section 4, we provide an exact formula for the bound, and also generalize the result in case players have unequal maximum achievable utilities. The former case, however, (equal maximum achievable utilities) is particularly important, since utility levels of different players are commonly normalized, so as the intercomparison of utilities between them becomes meaningful [13].

Figure 1 depicts the bound on the price of fairness described above. The graph illustrates the dependence of the bound on the number of players, for different values of $\alpha$ and in case all players have equal maximum achievable utilities.

Our results capture special cases presented in the literature. In particular, in [4] it is shown that the price of proportional fairness (i.e., for $\alpha=1$ ) is bounded by $1-\Theta\left(n^{-1 / 2}\right)$, and the price of max-min fairness (i.e., for $\alpha \rightarrow \infty)$ is bounded by $1-\Theta\left(n^{-1}\right)$. Both those results are compatible with (2); Section 4 includes more technical details.

Finally, we are able to construct examples that achieve a price of fairness very close to the bounds. The structure of the examples is simple and not pathological by any means; it corresponds to the case of having two types of players, with one consuming resources at a higher rate than the other to derive a unit of utility. More details are included in Section 5.

The results of this paper are particularly important since they

- can be used by a central decision maker in order to make a quantitative decision on which value of $\alpha$ to choose, in case she decides to employ the $\alpha$-fairness scheme and would like a guarantee on the maximum possible efficiency loss, 


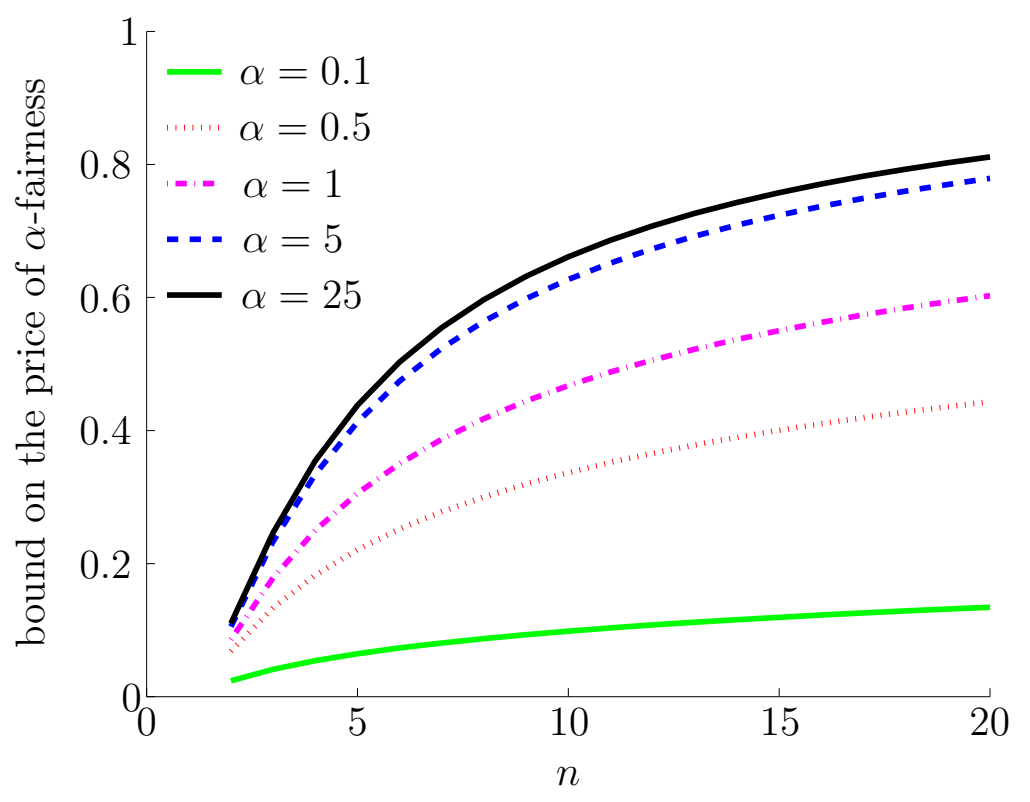

Figure 1: Bounds on the price of $\alpha$-fairness for different values of $\alpha$ in case players have equal maximum achievable utilities. The bounds are plotted as functions of the number of players $n$.

- shed light on the principle that a higher value of $\alpha$ (in most cases) incurs a higher efficiency loss,

- illustrate that the efficiency loss is very small for a small number of players $n$, and/or a small value of $\alpha$,

- allow one to identify near worst-case examples (see Section [5),

- answer the question of how the price of fairness is affected by different parameters. In particular, a crude analysis suggests that a higher value of $\alpha$, a higher value of number of players and asymmetry in the way players utilize the available resources all possibly result in higher efficiency loss. The bounds on the price of fairness can be used to better understand the dependence/sensitivity of the efficiency loss on those parameters.

\section{The price of fairness}

We present the results of this paper, namely near-tight bounds on the price of $\alpha$-fairness, in more detail. In the following subsections, we illustrate how this work connects with results in the literature on the important special cases of proportional and max-min fairness, by providing a unifying characterization of the price of fairness. Finally, we discuss properties of the bounds and auxiliary results.

For $\alpha=0$, the $\alpha$-fairness scheme corresponds to the utilitarian principle, since $W_{0}$ is the sum of utilities, $W_{0}(u)=\mathbf{1}^{T} u$. Hence, for any utility set $U$ that satisfies Assumption 1, the efficiency of the system is the same under both schemes, i.e., $\operatorname{SYSTEM}(U)=\operatorname{FAIR}(U ; 0)$, 
and

$$
\operatorname{POF}(U ; 0)=0 \text {. }
$$

We define a parameterized function that will be useful for the theorems presented below. For any $\alpha>0, n \in \mathbf{N} \backslash\{0,1\}$ and $r \geq 1$, let $f_{1}:[1, n] \rightarrow \mathbf{R}$ be defined as

$$
f_{1}(x ; \alpha, n, r)=\frac{r^{\frac{1}{\alpha}} x^{1+\frac{1}{\alpha}}+n-x}{r^{\frac{1}{\alpha}} x^{1+\frac{1}{\alpha}}+(n-x) r x} .
$$

In Section 4.2.1 we show that $-f_{1}$ is unimodal $^{1}$ over $[1, n]$ for any $\alpha>0$.

We now present the first main result. Recall that the maximum achievable utility of player $j$, is defined as

$$
u_{j}^{\star}=\sup \left\{u_{j} \mid u \in U\right\} \text {, for all } j=1, \ldots, n .
$$

Theorem 1. Consider a resource allocation problem with $n$ players, $n \geq 2$. Let the utility set, denoted by $U \subset \mathbf{R}_{+}^{n}$, be compact and convex. If the players have equal maximum achievable utilities (greater than zero) and $\alpha>0$, the price of $\alpha$-fairness is bounded by

$$
\operatorname{POF}(U ; \alpha) \leq 1-f_{1}\left(\xi_{1}^{\star} ; \alpha, n, 1\right),
$$

where

$$
f_{1}(x ; \alpha, n, 1)=\frac{x^{1+\frac{1}{\alpha}}+n-x}{x^{1+\frac{1}{\alpha}}+(n-x) x},
$$

and $\xi_{1}^{\star}$ is the unique value at which $f_{1}(. ; \alpha, n, 1)$ attains its minimum over $[1, n]$.

Note that since $-f_{1}$ is differentiable and unimodal over $[1, n]$ (by Proposition 21), the above bound can be routinely calculated. The proof of Theorem 1 is omitted, as it is a special case of the more general Theorem 2 that follows, in which we relax the constraint of equal maximum achievable utilities.

Bounds implied by Theorem 1 are shown in Figure 1, for different values of $\alpha$ and as functions of the number of players $n$.

In Section 4.2.1 we present a result that illustrates the impact of the inequality aversion parameter $\alpha$ on the price of fairness in a more clear way. In particular, we show that under the setup of Theorem 1, the price of $\alpha$-fairness is bounded by

$$
\operatorname{POF}(U ; \alpha) \leq 1-\Theta\left(n^{-\frac{\alpha}{\alpha+1}}\right) .
$$

We now relax the constraint in Theorem 1 of all players having equal maximum achievable utilities. Similarly to Theorem 1, we first define a parameterized function that will be useful to present the result below. For any $\alpha \in(0,1), n \in \mathbf{N} \backslash\{0,1\}$ and $B>0$, let $f_{2}: \mathbf{R}_{+} \rightarrow \mathbf{R}$ be defined as

$$
f_{2}(x ; \alpha, n, B)=\frac{1}{B}\left(x+(n-1)^{-\frac{\alpha}{1-\alpha}}\left(B x^{-\alpha}-x^{1-\alpha}\right)^{\frac{1}{1-\alpha}}\right) .
$$

In Section 4.2.1, we show that $f_{2}$ is convex.

\footnotetext{
${ }^{1}$ For $a, b \in \mathbf{R}$ with $a \leq b$, a function $f:[a, b] \rightarrow \mathbf{R}$ is unimodal if for some value $m$, it is strictly increasing for $a \leq x \leq m$ and strictly decreasing for $m \leq x \leq b$. The maximum of $f$ over $[a, b]$ is $f(m)$.
} 
Theorem 2. Consider a resource allocation problem with $n$ players, $n \geq 2$. Let the utility set, denoted by $U \subset \mathbf{R}^{n}$, be compact and convex. If the maximum achievable utilities of the players, $u_{1}^{\star}, \ldots, u_{n}^{\star}$, satisfy

$$
L \leq \min _{j=1, \ldots, n} u_{j}^{\star} \leq \max _{j=1, \ldots, n} u_{j}^{\star}=B
$$

for some $0<L \leq B$, the price of $\alpha$-fairness is bounded by

$$
\operatorname{POF}(U ; \alpha) \leq \begin{cases}1-\min \left\{f_{1}\left(\xi_{1}^{\star} ; \alpha, n, \frac{B}{L}\right), f_{2}\left(\xi_{2}^{\star} ; \alpha, n, B\right)\right\}, & \text { if } 0<\alpha<1, \\ 1-f_{1}\left(\xi_{1}^{\star} ; \alpha, n, \frac{B}{L}\right), & \text { if } \alpha \geq 1,\end{cases}
$$

where $\xi_{1}^{\star}$ is the unique value at which $f_{1}$ attains its minimum over $[1, n]$, and $\xi_{2}^{\star}$ a value at which $f_{2}$ attains its minimum over $\left[\frac{B}{n}, \frac{B}{\left(\frac{L}{B}\right)^{\frac{1-\alpha}{\alpha}}(n-1)+1}\right]$.

Proof. Without loss of generality, we assume that $U$ is monotone ${ }^{2}$. This is because both schemes we consider, namely utilitarian and $\alpha$-fairness yield Pareto optimal allocations. In particular, suppose there exist allocations $a \in U$ and $b \notin U$, with allocation $a$ dominating allocation $b$, i.e., $0 \leq b \leq a$. Note that allocation $b$ can thus not be Pareto optimal. Then, we can equivalently assume that $b \in U$, since $b$ cannot be selected by any of the schemes.

Note that the monotonicity assumption and the fact that the $j$ th player has a maximum achievable utility of $u_{j}^{\star}$ also imply that $u_{j}^{\star} e_{j} \in U$, for all $j=1, \ldots, n$.

Fix $\alpha>0$ and let $z=z(\alpha) \in U$ be the unique allocation under the $\alpha$-fairness criterion ( since $W_{\alpha}$ is strictly concave for $\alpha>0$ ), and assume, without loss of generality, that

$$
z_{1} \geq z_{2} \geq \ldots \geq z_{n}
$$

The necessary first order condition for the optimality of $z$ can be expressed as

$$
\nabla W_{\alpha}(z)^{T}(u-z) \leq 0 \Rightarrow \sum_{j=1}^{n} z_{j}^{-\alpha}\left(u_{j}-z_{j}\right) \leq 0, \quad \forall u \in U
$$

or equivalently

$$
\gamma^{T} u \leq 1, \quad \forall u \in U
$$

where

$$
\gamma_{j}=\frac{z_{j}^{-\alpha}}{\sum_{i} z_{i}^{1-\alpha}}, \quad j=1, \ldots, n
$$

Note that (3) implies

$$
\gamma_{1} \leq \gamma_{2} \leq \ldots \leq \gamma_{n}
$$

Also, since $u_{j}^{\star} e_{j} \in U$ for all $j$, (4) yields that

$$
u_{j}^{\star} \gamma_{j}=\gamma^{T}\left(u_{j}^{\star} e_{j}\right) \leq 1 \Rightarrow \gamma_{j} \leq \frac{1}{u_{j}^{\star}}, \quad j=1, \ldots, n .
$$

\footnotetext{
${ }^{2} \mathrm{~A}$ set $A \subset \mathbf{R}_{+}^{n}$ is called monotone if $\left\{b \in \mathbf{R}^{n} \mid 0 \leq b \leq a\right\} \subset A, \forall a \in A$, where the inequality sign notation for vectors is used for componentwise inequality.
} 
We now use (4), and the fact that each player has a maximum achievable utility to bound the system efficiency under the utilitarian principle as follows:

$$
\begin{aligned}
\operatorname{SYSTEM}(U) & =\max \left\{\mathbf{1}^{T} u \mid u \in U\right\} \\
& \leq \max \left\{\mathbf{1}^{T} u \mid 0 \leq u \leq u^{\star}, \gamma^{T} u \leq 1\right\} .
\end{aligned}
$$

Using the above inequality,

$$
\begin{aligned}
\operatorname{POF}(U ; \alpha) & =\frac{\operatorname{SYSTEM}(U)-\operatorname{FAIR}(U ; \alpha)}{\operatorname{SYSTEM}(U)} \\
& =1-\frac{\operatorname{FAIR}(U ; \alpha)}{\operatorname{SYSTEM}(U)} \\
& =1-\frac{\sum_{j=1}^{n} z_{j}}{\operatorname{SYSTEM}(U)} \\
& \leq 1-\frac{\sum_{j=1}^{n} z_{j}}{\max \left\{\mathbf{1}^{T} u \mid 0 \leq u \leq u^{\star}, \gamma^{T} u \leq 1\right\}}
\end{aligned}
$$

For a fixed allocation $z$, the optimal value of the optimization problem in the bound above is componentwise nondecreasing in $u^{\star}$, since the feasible set expands for higher values of $u^{\star}$. Thus, to obtain the wost-case bound we consider the case in which $u_{j}^{\star}$ attains its highest value possible, for every $j=1, \ldots, n$, and satisfy the assumptions of the Theorem. Since $u_{j}^{\star} \leq B$ and $u_{j}^{\star} \leq \frac{1}{\gamma_{j}}$ (by ([6]) ), we deal with the problem with maximum achievable utilities $\bar{u}^{\star}$, such that

$$
\bar{u}_{j}^{\star}=\min \left\{B, \frac{1}{\gamma_{j}}\right\}, \quad j=1, \ldots, n,
$$

and

$$
L \leq \min _{j=1, \ldots, n} \bar{u}_{j}^{\star} \leq \max _{j=1, \ldots, n} \bar{u}_{j}^{\star}=B .
$$

The optimization problem in (8) is the linear relaxation of the well-studied knapsack problem, a version of which we review next. Let $v \in \mathbf{R}_{+}^{n}$ and $w \in \mathbf{R}_{+}^{n}$ be such that $0<w_{1} \leq \ldots \leq w_{n}$, and $w_{1} v_{1} \leq 1$. Then, one can show (see [3]) that the linear optimization problem

$$
\begin{array}{ll}
\operatorname{maximize} & \mathbf{1}^{T} y \\
\text { subject to } & w^{T} y \leq 1 \\
& 0 \leq y \leq v
\end{array}
$$

has an optimal value equal to $\sum_{j=1}^{\ell(v, w)} v_{j}+\delta(v, w)$, where

$$
\begin{aligned}
& \ell(v, w)=\max \left\{i \mid \sum_{j=1}^{i} w_{j} v_{j} \leq 1, i \leq n-1\right\} \in\{1, \ldots, n-1\} \\
& \delta(v, w)=\frac{1-\sum_{j=1}^{\ell(v, w)} w_{j} v_{j}}{w_{\ell(v, w)+1}} \in\left[0, v_{\ell(v, w)+1}\right] .
\end{aligned}
$$


We can apply the above result to compute the optimal value of the problem in (8) , since $\gamma$ and the selected $\bar{u}^{\star}$ satisfy (5) and (6). Then,

$$
\max \left\{\mathbf{1}^{T} u \mid 0 \leq u \leq \bar{u}^{\star}, \gamma^{T} u \leq 1\right\}=\sum_{j=1}^{\ell\left(\bar{u}^{\star}, \gamma\right)} \bar{u}_{j}^{\star}+\delta\left(\bar{u}^{\star}, \gamma\right)
$$

In the above expression, $\bar{u}_{1}^{\star}$ is equal to $B$ (as the largest component of $\bar{u}^{\star}$ ). Moreover, all the remaining components of $\bar{u}^{\star}$ in the summation are also equal to $B$. To see this, note that by the definition of $\ell$ we get

$$
\sum_{j=1}^{\ell\left(\bar{u}^{\star}, \gamma\right)} \gamma_{j} \bar{u}_{j}^{\star} \leq 1
$$

Suppose that $\bar{u}_{i}^{\star}$ is not equal to $B$ for some $1<i \leq \ell\left(\bar{u}^{\star}, \gamma\right)$. By (9) it follows that $\bar{u}_{i}^{\star}=\frac{1}{\gamma_{i}}$, and

$$
\sum_{j=1}^{\ell\left(\bar{u}^{\star}, \gamma\right)} \gamma_{j} \bar{u}_{j}^{\star}>\gamma_{i} \bar{u}_{i}^{\star}=\gamma_{i} \frac{1}{\gamma_{i}}=1,
$$

a contradiction. Then, (13) can be further simplified to

$$
\max \left\{\mathbf{1}^{T} u \mid 0 \leq u \leq \bar{u}^{\star}, \gamma^{T} u \leq 1\right\}=B \ell\left(\bar{u}^{\star}, \gamma\right)+\delta\left(\bar{u}^{\star}, \gamma\right)
$$

The bound from (8) can now be rewritten,

$$
\operatorname{POF}(U ; \alpha) \leq 1-\frac{\sum_{j=1}^{n} z_{j}}{B \ell\left(\bar{u}^{\star}, \gamma\right)+\delta\left(\bar{u}^{\star}, \gamma\right)} .
$$

Consider the set $S$ in the $(n+3)$-dimensional space, defined by the following constraints with variables $d \in \mathbf{R}, \lambda \in \mathbf{N}$ and $x_{1}, \ldots, x_{\lambda}, \bar{x}_{\lambda+1}, \underline{x}_{\lambda+1}, x_{\lambda+2}, \ldots, x_{n} \in \mathbf{R}$ :

$$
\begin{aligned}
& 0 \leq d \leq 1 \\
& 1 \leq \lambda \leq n-1 \\
& 0 \leq x_{n} \leq \ldots \leq x_{\lambda+2} \leq \underline{x}_{\lambda+1} \leq \bar{x}_{\lambda+1} \leq x_{\lambda} \leq \ldots \leq x_{1} \leq B \\
& L x_{n}^{-\alpha} \leq x_{1}^{1-\alpha}+\ldots+x_{\lambda}^{1-\alpha}+d \bar{x}_{\lambda+1}^{1-\alpha}+(1-d) \underline{x}_{\lambda+1}^{1-\alpha}+x_{\lambda+2}^{1-\alpha}+\ldots+x_{n}^{1-\alpha} \\
& B\left(x_{1}^{-\alpha}+\ldots+x_{\lambda}^{-\alpha}+d \bar{x}_{\lambda+1}^{-\alpha}\right) \leq \\
& \quad \quad x_{1}^{1-\alpha}+\ldots+x_{\lambda}^{1-\alpha}+d \bar{x}_{\lambda+1}^{1-\alpha}+(1-d) \underline{x}_{\lambda+1}^{1-\alpha}+x_{\lambda+2}^{1-\alpha}+\ldots+x_{n}^{1-\alpha} .
\end{aligned}
$$

We show that

$$
\frac{\sum_{j=1}^{n} z_{j}}{B \ell\left(\bar{u}^{\star}, \gamma\right)+\delta\left(\bar{u}^{\star}, \gamma\right)} \geq \min _{(d, \lambda, x) \in S} \frac{x_{1}+\ldots+x_{\lambda}+d \bar{x}_{\lambda+1}+(1-d) \underline{x}_{\lambda+1}+x_{\lambda+2}+\ldots+x_{n}}{B \lambda+B d} .
$$

We pick values for $d, \lambda$ and $x$ that are such that (a) they are feasible for $S$, and (b) the function argument of the minimum, if evaluated at $(d, \lambda, x)$, is equal to the left-hand side of (16). In particular, let

$$
\begin{aligned}
d & =\frac{\delta\left(\bar{u}^{\star}, \gamma\right)}{B}, & \lambda & =\ell\left(\bar{u}^{\star}, \gamma\right), \\
x_{j} & =z_{j}, \quad j \neq \lambda+1, & \bar{x}_{\lambda+1} & =\underline{x}_{\lambda+1}=z_{\lambda+1} .
\end{aligned}
$$


Then, (15a), (15b) and (15c) are satisfied because of (12), (11) and (3) respectively. By the definition of $\gamma$ and the selected value of $x$, (15d) can be equivalently expressed as

$$
\gamma_{n} \leq \frac{1}{L}
$$

which is implied by $\bar{u}_{j}^{\star} \geq L$ and (5). Similarly, (15e) is equivalent to

$$
B \gamma_{1}+\ldots+B \gamma_{\ell\left(\bar{u}^{\star}, \gamma\right)}+\delta\left(\bar{u}^{\star}, \gamma\right) \gamma_{\ell\left(\bar{u}^{\star}, \gamma\right)+1} \leq 1
$$

which again holds true (by (12)). The function argument of the minimum, evaluated to the selected point, is clearly equal to the left-hand side of (16). Finally, the minimum is attained by the Weierstrass Theorem, since the function argument is continuous, and $S$ is compact. Note that (15d) in conjunction with (15c) bound $x_{n}$ away from 0 . In particular, if $\alpha \geq 1$, we get

$$
L x_{n}^{-\alpha} \leq x_{1}^{1-\alpha}+\ldots+x_{n}^{1-\alpha} \leq n x_{n}^{1-\alpha} \Rightarrow x_{n} \geq \frac{L}{n} .
$$

Similarly, for $\alpha<1$ we get

$$
x_{n} \geq\left(\frac{L}{n}\right)^{\frac{1}{\alpha}} B^{\frac{\alpha-1}{\alpha}} .
$$

To evaluate the minimum in (16), one can assume without loss of generality that for a point $\left(d^{\prime}, \lambda^{\prime}, x^{\prime}\right) \in S$ that attains the minimum, we have

$$
x_{1}^{\prime}=\ldots=x_{\lambda}^{\prime}=\bar{x}_{\lambda+1}^{\prime}, \quad \underline{x}_{\lambda+1}^{\prime}=x_{\lambda+2}^{\prime}=\ldots=x_{n}^{\prime} .
$$

Technical details are included in Section 4.2.2. Using this observation, we can further simplify (16). In particular, consider the set $T \subset \mathbf{R}^{3}$, defined by the following constraints (with variables $x_{1}, x_{2}$ and $\left.y\right)$ :

$$
\begin{aligned}
& 0 \leq x_{2} \leq x_{1} \leq B \\
& 1 \leq y \leq n \\
& L x_{2}^{-\alpha} \leq y x_{1}^{1-\alpha}+(n-y) x_{2}^{1-\alpha} \\
& B y x_{1}^{-\alpha} \leq y x_{1}^{1-\alpha}+(n-y) x_{2}^{1-\alpha} .
\end{aligned}
$$

We show that

$$
\min _{(d, \lambda, x) \in S} \frac{x_{1}+\ldots+x_{\lambda}+d \bar{x}_{\lambda+1}+(1-d) \underline{x}_{\lambda+1}+x_{\lambda+2}+\ldots+x_{n}}{B \lambda+B d} \geq \min _{\left(x_{1}, x_{2}, y\right) \in T} \frac{y x_{1}+(n-y) x_{2}}{B y} .
$$

Let $\left(d^{\prime}, \lambda^{\prime}, x^{\prime}\right) \in S$ be a point that attains the minimum of the left hand side above, satisfying (17). We construct a point $\left(x_{1}, x_{2}, y\right) \in T$, for which the objective of the minimum on the right hand side of (19) is equal to the minimum of the left hand side. Let $x_{1}=x_{1}^{\prime}, x_{2}=x_{n}^{\prime}$ and $y=\lambda^{\prime}+d^{\prime}$. Using (17) and the selected values for $x_{1}, x_{2}$ and $y$, we have that (15c) implies (18a) and that (15a - 15b) imply (18b). Similarly, (15d - 15e imply (18c - 18d) respectively. To show that the minimum of the right hand side of (19) is attained, one can use a similar argument as in showing (16). 
If we combine (14), (16), (19) we get

$$
\operatorname{POF}(U ; \alpha) \leq 1-\min _{\left(x_{1}, x_{2}, y\right) \in T} \frac{y x_{1}+(n-y) x_{2}}{B y} .
$$

The final step is the evaluation of the minimum above. Let $\left(x_{1}^{\star}, x_{2}^{\star}, y^{\star}\right) \in T$ be a point that attains the minimum. Then, we have

$$
y^{\star}<n, \quad x_{2}^{\star}<x_{1}^{\star} .
$$

To see this, suppose that $x_{2}^{\star}=x_{1}^{\star}$. Then, the minimum is equal to $\frac{n x_{1}^{\star}}{B y^{\star}}$. But, constraint (18d) yields that $n x_{1}^{\star} \geq B y^{\star}$, in which case the minimum is greater than or equal to 1 . Then, (20) yields that the price of fairness is always 0 , a contradiction. If $y^{\star}=n$, (18d $)$ suggests that $x_{1}^{\star}=B$. Also, the minimum is equal to $\frac{x_{1}^{\star}}{B}$, thus equal to 1 , a contradiction.

We now argue for $\alpha \geq 1$ and $\alpha<1$ separately.

$\alpha \geq 1$ : Suppose that both $(18 \mathrm{c}, 18 \mathrm{~d})$ are inactive at $\left(x_{1}^{\star}, x_{2}^{\star}, y^{\star}\right)$. Then, a small enough reduction in the value of $x_{2}^{\star}$ preserves feasibility (with respect to $T$ ), and also yields a strictly lower value for the minimum (since $y^{\star}<n$, by (21)), thus contradicting that the point attains the minimum. If $\alpha=1$ and one of (18c, $18 \mathrm{~d}$ ) is inactive, the right-hand side of both is equal to $n$. Then, we can simply reduce the variable that figures in the inactive constraint, preserving feasibility, and achieving a strictly lower value for the minimum. If $\alpha>1$, then we can jointly reduce $x_{1}^{\star}$ and $x_{2}^{\star}$, possibly by different amounts, such that the active of the two constraints remains active. We again have preservation of feasibility (since also $0<x_{2}^{\star}<x_{1}^{\star}$, by (21)) for small enough reductions, and a strictly lower value for the minimum. It follows that $\left(x_{1}^{\star}, x_{2}^{\star}, y^{\star}\right)$ satisfies (18c) and (18d) with equality.

Since for any point that attains the minimum constraints (18c-18d) are active, we can use the corresponding equations to solve for $x_{1}$ and $x_{2}$. We get

$$
\begin{aligned}
& x_{1}=\frac{\left(\frac{y B}{L}\right)^{\frac{1}{\alpha}} L}{n-y+y^{\frac{1}{\alpha}}\left(\frac{B}{L}\right)^{\frac{1}{\alpha}-1}} \\
& x_{2}=\frac{L}{n-y+y^{\frac{1}{\alpha}}\left(\frac{B}{L}\right)^{\frac{1}{\alpha}-1}} .
\end{aligned}
$$

Note that for $1 \leq y \leq n$, we clearly have that $0 \leq x_{2} \leq x_{1} \leq B$. Also, the objective of the minimum evaluates to

$$
\frac{\left(\frac{B}{L}\right)^{\frac{1}{\alpha}} y^{1+\frac{1}{\alpha}}+n-y}{\left(\frac{B}{L}\right)^{\frac{1}{\alpha}} y^{1+\frac{1}{\alpha}}+(n-y)\left(\frac{B}{L}\right) y}=f_{1}\left(y ; \alpha, n, \frac{B}{L}\right),
$$

if we substitute for $x_{1}, x_{2}$. Thus, (20) can be rewritten as

$$
\operatorname{POF}(U ; \alpha) \leq 1-\min _{\left(x_{1}, x_{2}, y\right) \in T} \frac{y x_{1}+(n-y) x_{2}}{B y}=1-f_{1}\left(\xi_{1}^{\star} ; \alpha, n, \frac{B}{L}\right) .
$$

Note that $\xi_{1}^{\star}$ is unique, since by Proposition $2,-f_{1}$ is unimodal. 
$\alpha<1$ : Suppose that $(18 \mathrm{~d})$ is inactive at $\left(x_{1}^{\star}, x_{2}^{\star}, y^{\star}\right)$. Then, we can increase $y^{\star}$ by a small positive value $\epsilon$, such that $(18 \mathrm{~d})$ and $(18 \mathrm{~b})$ are still satisfied. Constraint (18c) is then relaxed, since $\left(x_{1}^{\star}\right)^{1-\alpha}>\left(x_{2}^{\star}\right)^{1-\alpha}$. The minimum then has a strictly lower value, a contradiction. Hence, (18d) is active at any point that attains the minimum.

We now show that at least one of the following is true: $y^{\star}=1$, or (18c) is active at $\left(x_{1}^{\star}, x_{2}^{\star}, y^{\star}\right)$. Since (18d) holds with equality, we can solve for $y$ and substitute back its value. The objective of the minimum then becomes

$$
\frac{x_{1}+x_{2}^{\alpha}\left(B x_{1}^{-\alpha}-x_{1}^{1-\alpha}\right)}{B}
$$

and the constraints defining the set $T$ simplify to

$$
\begin{aligned}
& 0 \leq x_{2} \leq x_{1} \leq B \\
& B x_{1}^{-\alpha}-x_{1}^{1-\alpha}+x_{2}^{1-\alpha} \leq n x_{2}^{1-\alpha} \\
& B x_{1}^{-\alpha}-x_{1}^{1-\alpha}+x_{2}^{1-\alpha} \leq n \frac{B}{L} x_{1}^{-\alpha} x_{2} .
\end{aligned}
$$

In particular, constraints (26b) and (26c) correspond to constraints $y \geq 1$ and (18c) accordingly. Note that if $x_{2}=0$ at a point that attains the minimum, we get $x_{1} \geq B$ from (26b) and the minimum is equal to $\frac{x_{1}}{B} \geq 1$, a contradiction. If both (26b-26c) are not active at a point that attains the minimum, we can simply reduce $x_{2}$ by a small amount to preserve feasibility and achieve a strictly lower objective, a contradiction.

If (18c) is active, i.e., (26c) also, then we recover the case analyzed above for $a \geq 1$; in particular, $x_{1}$ and $x_{2}$ take the form as in (22) 23$)$, and the price of fairness is bounded as in (24).

If $y \geq 1$ is active, i.e., (26b) is active, we can solve for $x_{2}$ :

$$
x_{2}=(n-1)^{-\frac{1}{1-\alpha}}\left(B x_{1}^{-\alpha}-x_{1}^{1-\alpha}\right)^{\frac{1}{1-\alpha}},
$$

in which case the objective (25) is equal to $f_{2}\left(x_{1} ; \alpha, n, B\right)$. Similarly, constraints (26a) and (26c) become

$$
\begin{aligned}
& x_{1} \geq \frac{B}{n}, \text { and } \\
& x_{1} \leq \frac{B}{\left(\frac{L}{B}\right)^{\frac{1-\alpha}{\alpha}}(n-1)+1}
\end{aligned}
$$

respectively. Thus, (20) can be rewritten as

$$
\begin{aligned}
\operatorname{POF}(U ; \alpha) & \leq 1-\min _{\left(x_{1}, x_{2}, y\right) \in T} \frac{y x_{1}+(n-y) x_{2}}{B y} \\
& =1-\min _{\frac{B}{n} \leq x_{1} \leq \frac{\operatorname{lin}_{B}}{\left(\frac{L}{B}\right)^{\frac{1-\alpha}{\alpha}}(n-1)+1}} f_{2}\left(x_{1} ; \alpha, n, B\right) \\
& =1-f_{2}\left(\xi_{2}^{\star} ; \alpha, n, B\right) .
\end{aligned}
$$



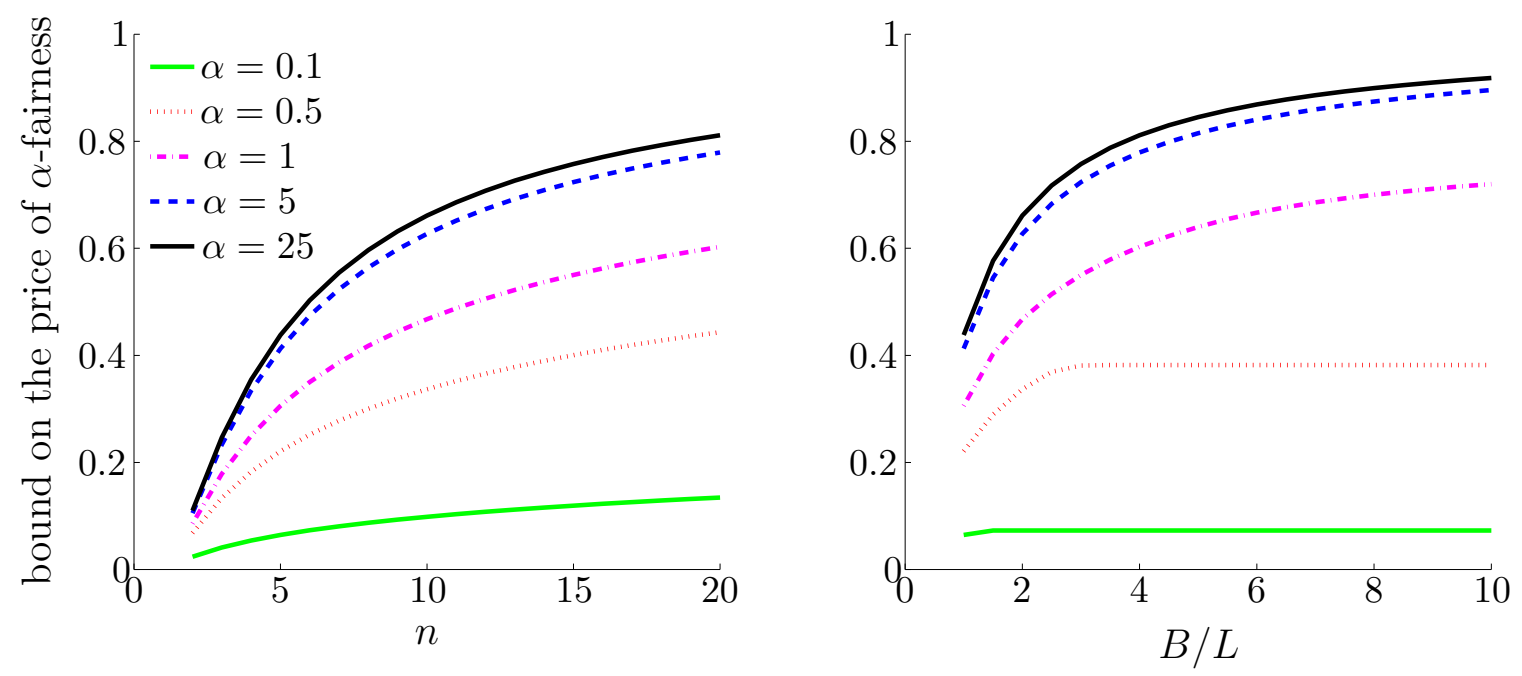

Figure 2: Bounds on the price of $\alpha$-fairness for different values of $\alpha$ as implied by Theorems 1, 2. On the left, players have equal maximum achievable utilities and the bounds are plotted as functions of the number of players $n$. On the right, the bounds are plotted as functions of $B / L$, for the case of $n=5$ players and $B=1$.

By combining the two cases for $\alpha<1$, we get

$$
\operatorname{POF}(U ; \alpha) \leq 1-\min \left\{f_{1}\left(\xi_{1}^{\star} ; \alpha, n, \frac{B}{L}\right), f_{2}\left(\xi_{2}^{\star} ; \alpha, n, B\right)\right\}
$$

Note that the result from Theorem 1 follows from the fact that for $L=B$ we get $\xi_{2}^{\star}=\frac{B}{n}$ and $f_{2}\left(\xi_{2}^{\star} ; \alpha, n, B\right)=1$.

Figure 2 depicts the bounds on the price of fairness implied by Theorems 1 and 2 , for some choices of the parameters they depend on. The graph on the left illustrates the dependence of the bound on the number of players, for different values of $\alpha$ and in case all players have equal maximum achievable utilities. The graph on the right, illustrates the dependence on potential asymmetry of the maximum achievable utilities (i.e., $B / L$ greater than 1 ), for different values of $\alpha$ and in case of $n=5$ players, $B=1$.

Note that one can very efficiently evaluate the bound on the price of fairness for a fixed $\alpha$, number of players $n$ and the bounds on the maximum achievable utilities of the players $B$ and $L$, since it involves only to evaluate the minima of at most two diffentiable functions over an interval, with one function being convex and the negative of the other being unimodal (see Proposition 2).

\subsection{The special cases of proportional and max-min fairness}

In this section, we show that our results capture special cases presented in the literature. In particular, the price of proportional and max-min fairness is studied in [4], primarily for the case of equal maximum achievable utilities. Since proportional and max-min fairness are both captured within the $\alpha$-fairness framework, for $\alpha=1$ and $\alpha \rightarrow \infty$ respectively (see Section 2), the price of proportional fairness is equal to $\operatorname{POF}(U ; 1)$, whereas we denote the 
price of max-min fairness with $\operatorname{POF}(U ; \infty)$. The results in [4] imply that, in case of equal maximum achievable utilities,

$$
\operatorname{POF}(U ; 1) \leq 1-\frac{2 \sqrt{n}-1}{n}, \quad \operatorname{POF}(U ; \infty) \leq 1-\frac{4 n}{(n+1)^{2}} .
$$

The following proposition shows that our analysis recovers those results as special cases.

Proposition 1. Consider a resource allocation problem with $n$ players, $n \geq 2$. Let the utility set, denoted by $U \subset \mathbf{R}^{n}$, be compact and convex. If the players have equal maximum achievable utilities (greater than zero),

$$
\operatorname{POF}(U ; 1) \leq 1-\frac{2 \sqrt{n}-1}{n} . \quad \text { (price of proportional fairness) }
$$

Let $\left\{\alpha_{k} \in \mathbf{R} \mid k \in \mathbf{N}\right\}$ be a sequence such that $\alpha_{k} \rightarrow \infty$ and $\alpha_{k} \geq 1, \forall k$. Then,

$$
\limsup _{k \rightarrow \infty} \operatorname{POF}\left(U ; \alpha_{k}\right) \leq 1-\frac{4 n}{(n+1)^{2}} . \quad \text { (price of max-min fairness) }
$$

Proof. Using Theorem 1 for $\alpha=1$ we get

$$
\begin{aligned}
\operatorname{POF}(U ; 1) & \leq 1-\min _{x \in[1, n]} f_{1}(x ; 1, n, 1) \\
& =1-\min _{x \in[1, n]} \frac{x^{2}+n-x}{n x} \\
& =1-\frac{2 \sqrt{n}-1}{n} .
\end{aligned}
$$

Similarly, for any $k \in \mathbf{N}$ and $\alpha=\alpha_{k}$

$$
\operatorname{POF}\left(U ; \alpha_{k}\right) \leq 1-\min _{x \in[1, n]} f_{1}\left(x ; \alpha_{k}, n, 1\right),
$$

which implies that

$$
\begin{aligned}
\limsup _{k \rightarrow \infty} \operatorname{POF}\left(U ; \alpha_{k}\right) & \leq \limsup _{k \rightarrow \infty}\left(1-\min _{x \in[1, n]} f_{1}\left(x ; \alpha_{k}, n, 1\right)\right) \\
& \leq 1-\liminf _{k \rightarrow \infty} \min _{x \in[1, n]} f_{1}\left(x ; \alpha_{k}, n, 1\right) .
\end{aligned}
$$

Consider the set of (real-valued) functions $\left\{f_{1}\left(. ; \alpha_{k}, n, 1\right) \mid k \in \mathbf{N}\right\}$ defined over the compact set $[1, n]$. We show that the set is equicontinuous, and that the closure of the set $\left\{f_{1}\left(x ; \alpha_{k}, n, 1\right) \mid k \in \mathbf{N}\right\}$ is bounded for any $x \in[1, n]$. Boundedness follows since $0 \leq$ $f_{1}(x ; \alpha, n, 1) \leq 1$ for any $\alpha>0$ and $x \in[1, n]$. The set of functions $\left\{f_{1}\left(. ; \alpha_{k}, n, 1\right) \mid k \in \mathbf{N}\right\}$ 
shares the same Lipschitz constant, as for any $k \in \mathbf{N}, \alpha_{k} \geq 1$ and $x \in[1, n]$ we have

$$
\begin{aligned}
\left|f_{1}^{\prime}\left(x ; \alpha_{k}, n, 1\right)\right| & =\left|\frac{\left(1-\frac{1}{\alpha_{k}}\right) x^{2+\frac{1}{\alpha_{k}}}+\frac{n+1}{\alpha_{k}} x^{1+\frac{1}{\alpha_{k}}}-n\left(1+\frac{1}{\alpha_{k}}\right) x^{\frac{1}{\alpha_{k}}}-(x-n)^{2}}{\left(x^{1+\frac{1}{\alpha_{k}}}+(n-x) x\right)^{2}}\right| \\
& \leq\left|\left(1-\frac{1}{\alpha_{k}}\right) x^{2+\frac{1}{\alpha_{k}}}+\frac{n+1}{\alpha_{k}} x^{1+\frac{1}{\alpha_{k}}}-n\left(1+\frac{1}{\alpha_{k}}\right) x^{\frac{1}{\alpha_{k}}}-(x-n)^{2}\right| \\
& \leq\left(1-\frac{1}{\alpha_{k}}\right) x^{2+\frac{1}{\alpha_{k}}}+\frac{n+1}{\alpha_{k}} x^{1+\frac{1}{\alpha_{k}}}+n\left(1+\frac{1}{\alpha_{k}}\right) x^{\frac{1}{\alpha_{k}}}+(x-n)^{2} \\
& \leq n^{3}+(n+1) n^{2}+2 n^{2}+n^{2}=2\left(n^{3}+2 n^{2}\right) .
\end{aligned}
$$

As a result, the set of functions $\left\{f_{1}\left(. ; \alpha_{k}, n, 1\right) \mid k \in \mathbf{N}\right\}$ is equicontinuous.

Using the above result,

$$
\lim _{k \rightarrow \infty} \min _{x \in[1, n]} f_{1}\left(x ; \alpha_{k}, n, 1\right)=\min _{x \in[1, n]} \lim _{k \rightarrow \infty} f_{1}\left(x ; \alpha_{k}, n, 1\right) .
$$

Thus, (28) yields

$$
\begin{aligned}
\limsup _{k \rightarrow \infty} \operatorname{POF}\left(U ; \alpha_{k}\right) & \leq 1-\liminf _{k \rightarrow \infty} \min _{x \in[1, n]} f_{1}\left(x ; \alpha_{k}, n, 1\right) \\
& =1-\min _{x \in[1, n]} \lim _{k \rightarrow \infty} f_{1}\left(x ; \alpha_{k}, n, 1\right) \\
& =1-\min _{x \in[1, n]} \lim _{k \rightarrow \infty} \frac{x^{1+\frac{1}{\alpha_{k}}}+n-x}{x^{1+\frac{1}{\alpha_{k}}}+(n-x) x} \\
& =1-\min _{x \in[1, n]} \frac{n}{x+(n-x) x} \\
& =1-\frac{4 n}{(n+1)^{2}}
\end{aligned}
$$

\subsection{Auxiliary results}

In this section, we provide technical details for the auxiliary results that were presented and used above without proof.

\subsubsection{Properties of the bounds}

First, we show that the parameterized functions, $f_{1}$ and $f_{2}$, used to present the main results have properties that allow one to efficiently calculate the bounds provided.

Proposition 2. Let $n \in \mathbf{N} \backslash\{0,1\}$. Then,

(a) $-f_{1}$ is unimodal over $[1, n]$, for any $\alpha>0$ and $r \geq 1$,

(b) $f_{2}$ is convex, for any $\alpha \in(0,1)$ and $B>0$. 
Proof. (a) We drop the parameters $\alpha, n$ and $r$ from the argument of $f_{1}$ to simplify notation. The derivative of $f_{1}$ is

$$
f_{1}^{\prime}(x)=\frac{g(x)}{\left(r^{\frac{1}{\alpha}} x^{1+\frac{1}{\alpha}}+(n-x) r x\right)^{2}}
$$

where

$$
g(x)=\left(1-\frac{1}{\alpha}\right) r^{1+\frac{1}{\alpha}} x^{2+\frac{1}{\alpha}}+\frac{r^{\frac{1}{\alpha}}}{\alpha}(n r+1) x^{1+\frac{1}{\alpha}}-n\left(1+\frac{1}{\alpha}\right) r^{\frac{1}{\alpha}} x^{\frac{1}{\alpha}}-r(x-n)^{2} .
$$

Note that the sign of the derivative is determined by $g(x)$, since the denominator is positive for $1 \leq x \leq n$, that is,

$$
\operatorname{sgn} f_{1}^{\prime}(x)=\operatorname{sgn} g(x) .
$$

We will show that $g$ is strictly increasing over $[1, n]$. To this end, we have

$$
g^{\prime}(x)=r^{\frac{1}{\alpha}} x^{\frac{1}{\alpha}-1} q(x)+2 r(n-x),
$$

where

$$
q(x)=\left(2+\frac{1}{\alpha}\right)\left(1-\frac{1}{\alpha}\right) r x^{2}+\left(1+\frac{1}{\alpha}\right)\left(\frac{n r+1}{\alpha}\right) x-\frac{n}{\alpha}\left(1+\frac{1}{\alpha}\right) .
$$

Since we are interested in the domain $[1, n]$, it suffices to show that $q(x)>0$ over it. For $\alpha>1, q$ is a convex quadratic, with its minimizer being equal to

$$
-\frac{\left(1+\frac{1}{\alpha}\right)\left(\frac{n r+1}{\alpha}\right)}{2\left(2+\frac{1}{\alpha}\right)\left(1-\frac{1}{\alpha}\right) r}<0 .
$$

Hence, $q(x) \geq q(1)$ for $x \in[1, n]$. Similarly, for $\alpha<1, q$ is a concave quadratic, and as such, for $x \in[1, n]$ we have $q(x) \geq \min \{q(1), q(n)\}$. For $\alpha=1, q(x)=2(n r+1) x-2 n$, which is positive for $x \geq 1$. Then, $q(x)>0$ in $[1, n]$ for all $\alpha>0$, if and only if $q(1)>0$ and $q(n)>0$. Note that for $r=1$, we get $q(1)=2$ and $q(n)=2 n^{2}$, and

$$
\frac{d q(1)}{d r}=2>0, \quad \frac{d q(n)}{d r}=2 n^{2}>0,
$$

which demonstrates that $q(1)$ and $q(n)$ are positive. Furthermore,

$$
g(n)=r^{\frac{1}{\alpha}} n^{1+\frac{1}{\alpha}}(r n-1)>0 .
$$

Using the above, the fact that $g$ is continuous and strictly increasing over $[1, n]$ and (29), we deduce that if $g(1)<0$, there exists a unique $m \in(1, n)$ such that

$$
\operatorname{sgn} f_{1}^{\prime}(x) \begin{cases}<0, & \text { if } 1 \leq x<m, \\ >0, & \text { if } m<x \leq n .\end{cases}
$$

Similarly, if $g(1) \geq 0, f_{1}$ is strictly increasing for $1 \leq x \leq n$. It follows that $-f_{1}$ is unimodal.

(b) For $\alpha \in(0,1)$ and $x>0, x^{-\alpha}$ is convex and $x^{1-\alpha}$ concave. It follows that $B x^{-\alpha}-x^{1-\alpha}$ is convex. Moreover, $x^{\frac{1}{1-\alpha}}$ is convex and nondecreasing. Hence, the composition of those two functions, $\left(B x^{-\alpha}-x^{1-\alpha}\right)^{\frac{1}{1-\alpha}}$, is convex (see Section 3.2.4 in [6] $)$. It follows that $f_{2}$ is convex. 
The next result deals with an asymptotic analysis of the bound implied by Theorem 11. The analysis illustrates the dependence of the bound on the risk aversion parameter $\alpha$ in a more clear way.

Proposition 3. Under the setup of Theorem 1, the price of $\alpha$-fairness is bounded by

$$
\operatorname{POF}(U ; \alpha) \leq 1-\Theta\left(n^{-\frac{\alpha}{\alpha+1}}\right) .
$$

Proof. Let $\theta_{n}=n^{\frac{\alpha}{\alpha+1}}$. Using the mean value Theorem, for every $n \geq 2$, there exists a $\psi_{n} \in\left[\theta_{n}, \xi_{1}^{\star}\right]$ (or $\left[\xi_{1}^{\star}, \theta_{n}\right]$, depending on if $\theta_{n} \leq \xi_{1}^{\star}$ ), such that

$$
f_{1}\left(\theta_{n} ; \alpha, n, 1\right)=f_{1}\left(\xi_{1}^{\star} ; \alpha, n, 1\right)+f_{1}^{\prime}\left(\psi_{n} ; \alpha, n, 1\right)\left(\theta_{n}-\xi_{1}^{\star}\right),
$$

or, equivalently,

$$
\frac{f_{1}\left(\xi_{1}^{\star} ; \alpha, n, 1\right)}{f_{1}\left(\theta_{n} ; \alpha, n, 1\right)}=1-\frac{f_{1}^{\prime}\left(\psi_{n} ; \alpha, n, 1\right)\left(\theta_{n}-\xi_{1}^{\star}\right)}{f_{1}\left(\theta_{n} ; \alpha, n, 1\right)} .
$$

We will show that, for a sufficiently small $\epsilon>0$

(a) $f_{1}^{\prime}\left(\psi_{n} ; \alpha, n, 1\right)=O\left(n^{-\frac{\min \{1, \alpha\}+2 \alpha}{\alpha+1}+2 \epsilon}\right)$,

(b) $\theta_{n}-\xi_{1}^{\star}=O\left(n^{\frac{\alpha}{\alpha+1}+\epsilon}\right)$,

(c) $f_{1}\left(\theta_{n} ; \alpha, n, 1\right)=\Theta\left(n^{-\frac{\alpha}{\alpha+1}}\right)$.

Using the above facts, it is easy to see that

$$
\frac{f_{1}\left(\xi_{1}^{\star} ; \alpha, n, 1\right)}{f_{1}\left(\theta_{n} ; \alpha, n, 1\right)}=1-\frac{f_{1}^{\prime}\left(\psi_{n} ; \alpha, n, 1\right)\left(\theta_{n}-\xi_{1}^{\star}\right)}{f_{1}\left(\theta_{n} ; \alpha, n, 1\right)}=1-O\left(n^{-\frac{\min \{1, \alpha\}}{\alpha+1}+3 \epsilon}\right) \rightarrow 1,
$$

and thus $f_{1}\left(\xi_{1}^{\star} ; \alpha, n, 1\right)=\Theta\left(n^{-\frac{\alpha}{\alpha+1}}\right)$, which will complete the proof.

(a) We first show that for any sufficiently large $n$,

$$
n^{\frac{\alpha}{\alpha+1}-\epsilon} \leq \xi_{1}^{\star} \leq n^{\frac{\alpha}{\alpha+1}+\epsilon} .
$$

By Proposition 2, $\xi_{1}^{\star}$ is the unique root of $g$ (defined therein) in the interval $[1, n]$. Moreover, $g$ is strictly increasing. The dominant term of

$$
\begin{aligned}
g\left(n^{\frac{\alpha}{\alpha+1}-\epsilon}\right)=\left(1-\frac{1}{\alpha}\right) n^{\left(2+\frac{1}{\alpha}\right)\left(\frac{\alpha}{\alpha+1}-\epsilon\right)} & +\frac{1}{\alpha} n^{1-\frac{\alpha+1}{\alpha} \epsilon}+\frac{1}{\alpha} n^{2-\frac{\alpha+1}{\alpha} \epsilon} \\
& -\left(1+\frac{1}{\alpha}\right) n^{1+\frac{1}{\alpha+1}-\frac{1}{\alpha} \epsilon}-n^{2}-n^{\frac{2 \alpha}{\alpha+1}-2 \epsilon}+2 n^{1+\frac{\alpha}{\alpha+1}-\epsilon},
\end{aligned}
$$

is $-n^{2}$, and hence, for sufficiently large $n$ we have $g\left(n^{\frac{\alpha}{\alpha+1}-\epsilon}\right)<0$. Similarly, the dominant term of $g\left(n^{\frac{\alpha}{\alpha+1}+\epsilon}\right)$ is $\frac{1}{\alpha} n^{2+\frac{\alpha+1}{\alpha}} \epsilon$, and for sufficiently large $n$ we have $g\left(n^{\frac{\alpha}{\alpha+1}+\epsilon}\right)>0$. The claim then follows. Using the above bound, for sufficiently large $n$, we also get that $\psi_{n} \geq n^{\frac{\alpha}{\alpha+1}-\epsilon}$. 
We now provide a bound for the denominator of $f_{1}^{\prime}\left(\psi_{n} ; \alpha, n, 1\right)$. In particular, for sufficiently large $n$, we get that for $x \leq n^{\frac{\alpha}{\alpha+1}+\epsilon}$,

$$
\frac{d}{d x}\left(x^{1+\frac{1}{\alpha}}+n x-x^{2}\right)=\left(1+\frac{1}{\alpha}\right) x^{\frac{1}{\alpha}}+n-2 x>0,
$$

which shows that the denominator is strictly increasing. Hence, using the lower bound on $\psi_{n}$,

$$
\begin{aligned}
\frac{1}{\left(\psi_{n}^{1+\frac{1}{\alpha}}+n \psi_{n}-\psi_{n}^{2}\right)^{2}} & \leq \frac{1}{\left(n^{\left(\frac{\alpha}{\alpha+1}-\epsilon\right)\left(1+\frac{1}{\alpha}\right)}+n^{1+\frac{\alpha}{\alpha+1}-\epsilon}-n^{\frac{2 \alpha}{\alpha+1}-2 \epsilon}\right)^{2}} \\
& \leq \frac{n^{-2-\frac{2 \alpha}{\alpha+1}+2 \epsilon}}{\left(n^{-\frac{\alpha}{\alpha+1}-\frac{1}{\alpha} \epsilon}+1-n^{-\frac{1}{\alpha+1}}\right)^{2}}=O\left(n^{-2-\frac{2 \alpha}{\alpha+1}+2 \epsilon}\right) .
\end{aligned}
$$

We now provide a bound for the numerator. Since $g$ is strictly increasing and $\xi_{1}^{\star}$ is a root, we get

$$
\begin{aligned}
\left|g\left(\psi_{n}\right)\right| & \leq\left|g\left(\theta_{n}\right)\right| \\
& =\left|\left(1-\frac{1}{\alpha}\right) \alpha^{\frac{2 \alpha+1}{\alpha+1}} n^{-\frac{1}{\alpha+1}+2}+n-\left(1+\frac{1}{\alpha}\right) \alpha^{\frac{1}{\alpha+1}} n^{-\frac{\alpha}{\alpha+1}+2}-\alpha^{\frac{2 \alpha}{\alpha+1}} n^{-\frac{2}{\alpha+1}+2}+2 \alpha^{\frac{\alpha}{\alpha+1}} n^{-\frac{1}{\alpha+1}+2}\right| \\
& =O\left(n^{-\frac{\min \{1, \alpha\}}{\alpha+1}+2}\right) .
\end{aligned}
$$

If we combine the above results, we get $f_{1}^{\prime}\left(\psi_{n} ; \alpha, n, 1\right)=O\left(n^{-\frac{\min \{1, \alpha\}+2 \alpha}{\alpha+1}+2 \epsilon}\right)$.

(b) Follows from (30).

(c) We have

$$
\begin{aligned}
f_{1}\left(\theta_{n} ; \alpha, n, 1\right) & =\frac{n+n-n^{\frac{\alpha}{\alpha+1}}}{n+n^{1+\frac{\alpha}{\alpha+1}}-n^{\frac{2 \alpha}{\alpha+1}}} \\
& =\frac{n\left(2-n^{-\frac{1}{\alpha+1}}\right)}{n^{1+\frac{\alpha}{\alpha+1}}\left(n^{-\frac{\alpha}{\alpha+1}}+1-n^{-\frac{1}{\alpha+1}}\right)}=\Theta\left(n^{-\frac{\alpha}{\alpha+1}}\right) .
\end{aligned}
$$

\subsubsection{Characterization of the central optimization problem in Theorem 2}

We conclude by providing technical details about the simplifying assumption (17), used in the proof of Theorem 2 .

Proposition 4. For a point $(d, \lambda, x) \in S$ that attains the minimum of (16)),

(a) if $\lambda+1<n$, then without loss of generality

$$
\underline{x}_{\lambda+1}=x_{\lambda+2}=\ldots=x_{n}, \text { and, }
$$

(b) without loss of generality

$$
x_{1}=\ldots=x_{\lambda}=\bar{x}_{\lambda+1} .
$$


Proof. (a) We drop the underline notation for $\underline{x}_{\lambda+1}$ to simplify notation. Suppose that $x_{j}>x_{j+1}$, for some index $j \in\{\lambda+1, \ldots, n-1\}$. We will show that there always exists a new point, $\left(d, \lambda, x^{\prime}\right) \in S$, for which $x_{i}^{\prime}=x_{i}$, for all $i \in\{1, \ldots, n\} \backslash\{j, j+1\}$, and which either achieves the same objective with $x_{j}^{\prime}=x_{j+1}^{\prime}$, or it achieves a strictly lower objective.

If $j=\lambda+1$ and $d=1$, we set $x_{j}^{\prime}=x_{j+1}^{\prime}=x_{j+1}$. The new point is feasible, and the objective attains the same value.

Otherwise, let $x_{j}^{\prime}=x_{j}-\epsilon$, for some $\epsilon>0$. We have two cases.

$\alpha \geq 1$ : Let $x_{j+1}^{\prime}=x_{j+1}$ and pick $\epsilon$ small enough, such that $x_{j}^{\prime} \geq x_{j+1}^{\prime}$. Moreover, for the new point (compared to the feasible starting point) the left-hand sides of (15d) and (15e) are unaltered, whereas the right-hand sides are either unaltered (for $\alpha=1$ ) or greater, since $x_{j}^{1-\alpha}<\left(x_{j}-\epsilon\right)^{1-\alpha}$ for $\alpha>1$. Hence, the new point is feasible. It also achieves a strictly lower objective value.

$\alpha<1$ : Let $x_{j+1}^{\prime}=x_{j+1}+\rho b \epsilon$, where

$$
\begin{aligned}
& b= \begin{cases}1-d, & \text { if } j=\lambda+1, \\
1, & \text { otherwise, }\end{cases} \\
& \rho \in\left(\frac{x_{j}^{-\alpha}}{x_{j+1}^{-\alpha}}, 1\right) .
\end{aligned}
$$

For $\epsilon$ small enough, we have $x_{j}^{\prime} \geq x_{j+1}^{\prime}$. For the new point, the left-hand side of (15d) either decreases (if $j+1=n$ ), or remains unaltered. The left-hand side of (15e) remains also unaltered. For the right-hand sides, since the only terms that change are those involving $x_{j}$ and $x_{j+1}$, we use a first order Taylor series expansion to get

$$
\begin{aligned}
b\left(x_{j}^{\prime}\right)^{1-\alpha}+\left(x_{j+1}^{\prime}\right)^{1-\alpha} & =b\left(x_{j}-\epsilon\right)^{1-\alpha}+\left(x_{j+1}+\rho b \epsilon\right)^{1-\alpha} \\
& =b x_{j}^{1-\alpha}-b \epsilon(1-\alpha) x_{j}^{-\alpha}+x_{j+1}^{1-\alpha}+\rho b \epsilon(1-\alpha) x_{j+1}^{-\alpha}+O\left(\epsilon^{2}\right) \\
& =\left(b x_{j}^{1-\alpha}+x_{j+1}^{1-\alpha}\right)+b(1-\alpha)\left(\rho x_{j+1}^{-\alpha}-x_{j}^{-\alpha}\right) \epsilon+O\left(\epsilon^{2}\right) .
\end{aligned}
$$

By the selection of $\rho$, the coefficient of the first order term (with respect to $\epsilon$ ) above is positive, and hence, for small enough $\epsilon$ we get

$$
b\left(x_{j}^{\prime}\right)^{1-\alpha}+\left(x_{j+1}^{\prime}\right)^{1-\alpha}>b x_{j}^{1-\alpha}+x_{j+1}^{1-\alpha} .
$$

That shows that the right hand side increases, and the new point is feasible. Finally, the difference in the objective value is $-b \epsilon+\rho b \epsilon$, and thus negative.

(b) We drop the overline notation for $\bar{x}_{\lambda+1}$ to simplify notation. Suppose that $x_{j}>x_{j+1}$, for some index $j \in\{1, \ldots, \lambda\}$.

We will show that there always exists a new point, $\left(d, \lambda, x^{\prime}\right) \in S$, for which $x_{i}^{\prime}=x_{i}$, for all $i \in\{1, \ldots, n\} \backslash\{j, j+1\}$, and which either achieves the same objective with $x_{j}^{\prime}=x_{j+1}^{\prime}$, or it achieves a strictly lower objective.

If $j+1=\lambda+1$ and $d=0$, we set $x_{j}^{\prime}=x_{j+1}^{\prime}=x_{j}$. The new point is feasible, and the objective attains the same value. 
Otherwise, let

$$
\begin{aligned}
x_{j}^{\prime} & =x_{j}-\epsilon \\
x_{j+1}^{\prime} & =x_{j+1}+\rho c \epsilon,
\end{aligned}
$$

for some $\epsilon>0$, where

$$
\begin{aligned}
& \rho \in\left(\frac{x_{j+1}}{x_{j}}, \frac{x_{j+1}^{-\alpha}}{x_{j}^{-\alpha}}\right) \\
& c=\frac{x_{j}^{-\alpha}}{b x_{j+1}^{-\alpha}} \\
& b= \begin{cases}d, & \text { if } j+1=\lambda+1, \\
1, & \text { otherwise }\end{cases}
\end{aligned}
$$

For $\epsilon$ small enough, we have $x_{j}^{\prime} \geq x_{j+1}^{\prime}$. For the new point, the left-hand side of (15d) remains unaltered. For the left-hand side of (15e) we use a first order Taylor series expansion (similarly as above) to get

$$
\begin{aligned}
\left(x_{j}^{\prime}\right)^{-\alpha}+b\left(x_{j+1}^{\prime}\right)^{-\alpha} & =\left(x_{j}-\epsilon\right)^{-\alpha}+b\left(x_{j+1}+\rho c \epsilon\right)^{-\alpha} \\
& =x_{j}^{-\alpha}+\epsilon \alpha x_{j}^{-\alpha-1}+b x_{j+1}^{-\alpha}-b \rho c \epsilon \alpha x_{j+1}^{-\alpha-1}+O\left(\epsilon^{2}\right) \\
& =\left(x_{j}^{-\alpha}+b x_{j+1}^{-\alpha}\right)+\epsilon \alpha x_{j}^{-\alpha-1}-\rho \epsilon \alpha x_{j}^{-\alpha} x_{j+1}^{-1}+O\left(\epsilon^{2}\right) \\
& =\left(x_{j}^{-\alpha}+b x_{j+1}^{-\alpha}\right)+\alpha x_{j}^{-\alpha-1}\left(1-\rho \frac{x_{j}}{x_{j+1}}\right) \epsilon+O\left(\epsilon^{2}\right) .
\end{aligned}
$$

By the selection of $\rho$, the coefficient of the first order term (with respect to $\epsilon$ ) above is negative, and hence, for small enough $\epsilon$ we get that the left-hand side decreases.

For the right-hand side of (15d) and (15e), we similarly get that

$$
\begin{aligned}
\left(x_{j}^{\prime}\right)^{1-\alpha}+b\left(x_{j+1}^{\prime}\right)^{1-\alpha} & =\left(x_{j}-\epsilon\right)^{1-\alpha}+b\left(x_{j+1}+\rho c \epsilon\right)^{1-\alpha} \\
& =x_{j}^{1-\alpha}-\epsilon(1-\alpha) x_{j}^{-\alpha}+b x_{j+1}^{1-\alpha}+b \rho c \epsilon(1-\alpha) x_{j+1}^{1-\alpha}+O\left(\epsilon^{2}\right) \\
& =\left(x_{j}^{1-\alpha}+b x_{j+1}^{1-\alpha}\right)+(1-\alpha) x_{j}^{-\alpha}(\rho-1) \epsilon+O\left(\epsilon^{2}\right) .
\end{aligned}
$$

If for $\alpha>1$ we pick $\rho<1$, and for $\alpha<1$ we pick $\rho>1$, the first order term (with respect to $\epsilon)$ above is positive, and hence, for small enough $\epsilon$ we get that the right-hand side increases for $\alpha \neq 1$. For $\alpha=1$, the right-hand side remains unaltered.

In all cases, the new point is feasible, and the difference in the objective value is

$$
-\epsilon+\rho c b \epsilon=(\rho c b-1) \epsilon=\left(\rho \frac{x_{j}^{-\alpha}}{x_{j+1}^{-\alpha}}-1\right) \epsilon,
$$

and thus negative (by the selection of $\rho$ ). 


\section{Near worst-case examples}

In this section, we demonstrate how one can construct near worst-case examples, for which the price of fairness is very close to the bounds implied by Theorems 1 and 2, for any values of the problem parameters; the number of players $n$, the value of the inequality aversion parameter $\alpha$, and the bounds on the maximum achievable utilities of the players, $B$ and $L$. In the following subsection, we provide an application for illustration purposes.

In [4], the authors demonstrate that, primarily for equal maximum achievable utilities, the bounds on the price of fairness are tight for the special cases of proportional fairness, if $\sqrt{n}$ is an integer, and for max-min fairness, for all $n$. In this work we deal with the more general case of $\alpha$-fairness and unequal maximum achievable utilities. The examples suggest that the bounds are very strong, near-tight. In particular, in the presented cases below, the bounds are greater than the actual price of fairness by at most 0.005 on average, and by 0.023 at most.

For any $n \in \mathbf{N} \backslash\{0,1\}, \alpha>0$ and $0<L \leq B$ we create a utility set using Procedure 1.

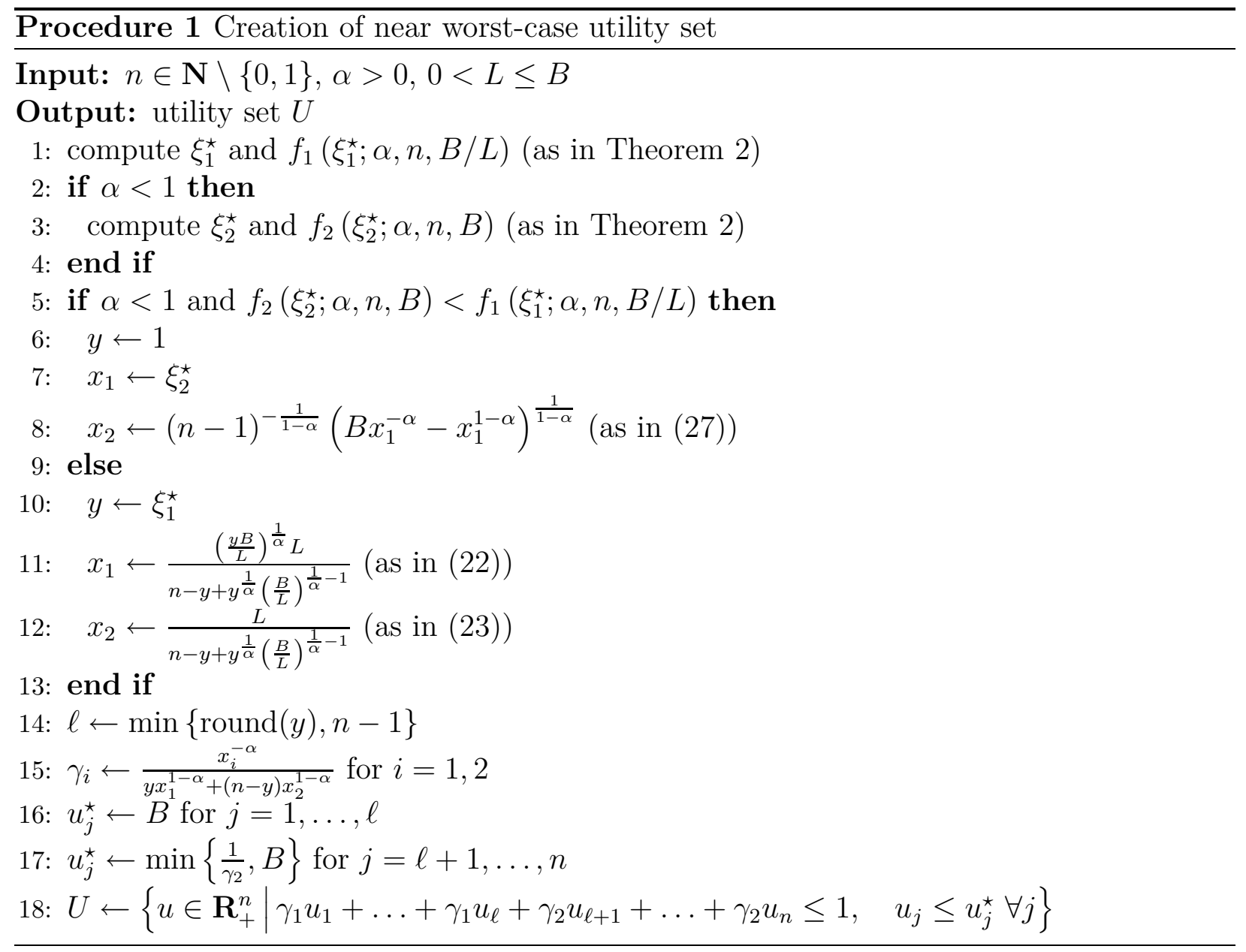

The following proposition demonstrates why Procedure 1 creates utility sets that achieve a price of fairness very close to the bounds implied by Theorem 2 . 
Proposition 5. For any $n \in \mathbf{N} \backslash\{0,1\}, \alpha>0,0<L \leq B$, the output utility set $U$ of Procedure 1 satisfies the conditions of Theorem 圆. If $y \in \mathbf{N}$, the output utility set $U$ satisfies the bound of Theorem 0 with equality.

Proof. The output utility set $U$ is a bounded polyhehron, hence convex and compact. Boundedness follows from positivity of $\gamma_{1}$ and $\gamma_{2}$.

Note that the selection of $x_{1}, x_{2}$ and $y$ in Procedure 1 corresponds to a point that attains the minimum of (201), hence all properties quoted in the proof of Theorem 2 apply. In particular, by (15d) we have $L \gamma_{2} \leq 1$ and (18d) is tight, $B y \gamma_{1}=1$. Moreover, the bound from Theorem 2 can be expressed as

$$
\operatorname{POF}(U ; \alpha) \leq 1-\frac{y x_{1}+(n-y) x_{2}}{B y} .
$$

The maximum achievable utility of the $j$ th player is equal to $u_{j}^{\star}$. To see this, note that the definition of $U$ includes the constraint $u_{j} \leq u_{j}^{\star}$, so it suffices to show that $u_{j}^{\star} e_{j} \in U$. For $j \leq \ell$, $u_{j}^{\star}=B$, and $\gamma_{1} B \leq \gamma_{1} y B=1$. For $j>\ell, u_{j}^{\star}=\min \left\{\frac{1}{\gamma_{2}}, B\right\}$ and $\gamma_{2} \min \left\{\frac{1}{\gamma_{2}}, B\right\} \leq \gamma_{2} \frac{1}{\gamma_{2}}=1$. Note also that $\min \left\{\frac{1}{\gamma_{2}}, B\right\} \geq L$, since $B \geq L$ and $L \gamma_{2} \leq 1$. It follows that $U$ satisfies the conditions of Theorem 2 .

Suppose that $y \in \mathbf{N}$. By (21) and the choice of $\ell$ in Procedure 1, we get $\ell=y$. Consider the vector $z \in \mathbf{R}^{n}$ with $z_{1}=\ldots=z_{\ell}=x_{1}$ and $z_{\ell+1}=\ldots=z_{n}=x_{2}$. Then, the sufficient first order optimality condition for $z$ to be the $\alpha$-fair allocation of $U$ is satisfied, as for any $u \in U$

$$
\sum_{j=1}^{n} z_{j}^{-\alpha}\left(u_{j}-z_{j}\right)=x_{1}^{-\alpha}\left(u_{1}+\ldots+u_{\ell}\right)+x_{2}^{-\alpha}\left(u_{\ell+1}+\ldots+u_{n}\right)-y x_{1}^{1-\alpha}-(n-y) x_{2}^{1-\alpha} \leq 0,
$$

since $\gamma_{1}\left(u_{1}+\ldots+u_{\ell}\right)+\gamma_{2}\left(u_{\ell+1}+\ldots+u_{n}\right) \leq 1$. Hence,

$$
\operatorname{FAIR}(U ; \alpha)=\mathbf{1}^{T} z=y x_{1}+(n-y) x_{2} .
$$

For the efficiency-maximizing solution, since $B y \gamma_{1}=1$, we get

$$
\operatorname{SYSTEM}(U)=B y \text {. }
$$

Then,

$$
\operatorname{POF}(U ; \alpha)=1-\frac{y x_{1}+(n-y) x_{2}}{B y},
$$

which is exactly the bound from Theorem 2 ,

The above result illustrates that one should expect Procedure 11 to generate examples that have a price of fairness very close to the established bounds. In particular, Proposition 5 shows that the source of error between the price of fairness for the utility sets generated by Procedure 1 and the bound is the (potential) non-integrality of $y$. In case that error is "large", one can search in the neighborhood of parameters $\gamma_{1}$ and $\gamma_{2}$ for an example that achieves a price closer to the bound, for instance by using finite-differencing derivatives and a gradient descent method (respecting feasibility). 

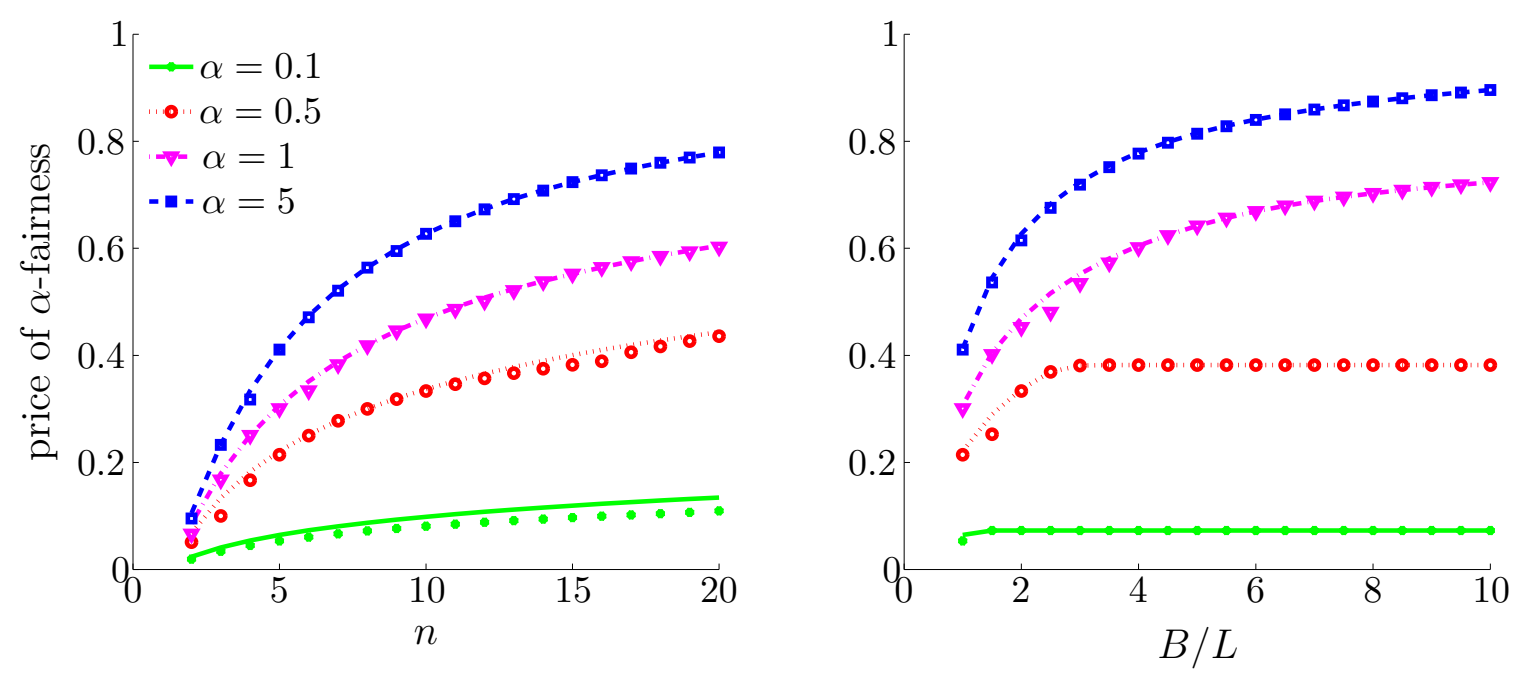

Figure 3: The price of $\alpha$-fairness for the examples of Section 5 (markers), for different values of $\alpha$. The corresponding bounds are also plotted (lines). On the left, players have equal maximum achievable utilities, $B=L$, and the values / bounds are plotted as functions of the number of players $n$. On the right, the values / bounds are plotted as functions of $B / L$, for the case of $n=5$ players and $B=1$.

Figure 3 illustrates the results of the above method. In particular, we use Procedure 1 to generate examples, and if the price is smaller than the bound minus 0.025 , we use a gradient descent method to get a better example. In both graphs, the lines illustrate the bounds, whereas the markers show the price of the generated utility set examples. The graph on the left deals with the case of all players having equal maximum achievable utilities, for different values of $\alpha$ and $n$. The graph on the right, deals with cases of assymetry of the maximum achievable utilities (i.e., $B / L$ greater than 1 ), for different values of $\alpha$ and in case of $n=5$ players, $B=1$. Out of the 152 examples depicted (as markers), the gradient descent improvement was employed 26 times. The average discrepancy between the bound and the values is 0.005 , and the largest discrepancy is 0.023 .

Finally, note that the structure of the near worst-case examples above pertains to a case of a single resource shared by $n$ players, who can be of two types. Players of the first type consume the resource at a lower rate, for a unit of utility, compared to players of the second type (the rates in the examples above are $\gamma_{1}$ and $\gamma_{2}$, respectively). In the next section, we further illustrate the structure of the worst-case examples for a problem of bandwidth allocation on a communication network

\subsection{Near worst-case bandwidth allocation}

We illustrate the structure of the worst-case examples for the price of $\alpha$-fairness via means of a bandwidth allocation problem in a network.

The network consists of hubs (nodes) that are connected via capacitated links (edges). Clients, or flows, wish to establish transmission from one hub to another over the network, via a pre-specified and fixed route. The network administrator needs to decide on the transmission rate assigned to each flow, subject to capacity constraints. The resources to be 


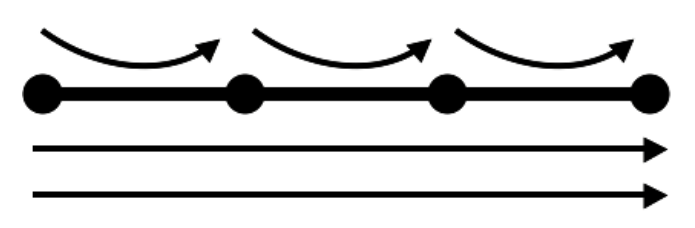

Figure 4: The network flow topology in case of $n=5$ and $y=3$, for the example in Section 5.1.

allocated in this case are the available bandwidth of the links, the players are the flows, and the central decision maker is the network administrator. The utility derived by each player is equal to his assigned transmission rate.

In the above setup, if all links have capacity of 1 , then, each player has a maximum achievable utility of 1 , which is trivially achieved if all other flows are assigned zero rates. Thus, Theorem 1 applies. Moreover, one can utilize Proposition 5 and Procedure 1 to construct near worst-case network topologies. In particular, one can show that the linegraph discussed below, actually corresponds to a worst-case topology in this setup.

Suppose that we fix the number of players $n \geq 2$, the desired inequality aversion parameter $\alpha>0$, and follow Procedure 1. Further suppose that $y \in \mathbf{N}$, as in Proposition 5 . Consider then a network with $y$ links of unit capacity, in a line-graph topology: the routes of the first $y$ flows are disjoint and they all occupy a single (distinct) link. The remaining $n-y$ flows have routes that utilize all $y$ links. The described network topology is shown in Figure 4, for $y=3$ and $n=5$. Each flow derives a utility equal to its assigned nonnegative rate, which we denote $u_{1}, \ldots, u_{n}$. We next show that the price of $\alpha$-fairness for this network is equal to the bound of Theorem 1.

The output utility set of Procedure 1 achieves the bound, by Proposition [5, since $y \in \mathbf{N}$. Moreover, since $B=L=1$ in this setting, we also get that $y \gamma_{1}=1$ and $\gamma_{2}=1$. Hence, the output utility set that achieves the bound can be formulated as

$$
U=\left\{u \geq 0 \mid u_{1}+\ldots+u_{y}+y\left(u_{y+1}+\ldots+u_{n}\right) \leq y, u \leq \mathbf{1}\right\}
$$

The utility set corresponding to the line-graph example above can be expressed using the nonnegativity constraints of the flow rates, and the capacity constraints on each of the $y$ links as follows,

$$
\bar{U}=\left\{u \geq 0 \mid u_{j}+u_{y+1}+\ldots+u_{n} \leq 1, j=1, \ldots, y\right\}
$$

Clearly, the maximum system efficiency under both sets is equal to $y$, simply by setting the first $y$ components of $u$ to 1 . It suffices then to show that the two sets also share the same $\alpha$-fair allocation. In particular, by symmetry of $U$ and strict concavity of $W_{\alpha}$, if $u^{F}$ is its $\alpha$ fair allocation, then $u_{1}^{F}=\ldots=u_{y}^{F}$, and $u_{y+1}^{F}=\ldots=u_{n}^{F}$. As a result, it follows that $u^{F} \in \bar{U}$. Finally, noting that all inequalities in the definition of $U$ are also valid for $\bar{U}$, it follows that $\bar{U} \subset U$ and that $u^{F}$ is also the $\alpha$-fair allocation of $\bar{U}$. 


\section{References}

[1] A. B. Atkinson. On the measurement of inequality. Journal of Economic Theory, 2(3): 244-263, 1970.

[2] N. Barr. The Economics of the Welfare State. Weidenfeld and Nicolson, 1987.

[3] D. Bertsimas and J. N. Tsitsiklis. Introduction to Linear Optimization. Athena Scientific, 1997.

[4] D. Bertsimas, V. F. Farias, and N. Trichakis. The price of fairness. Operations Research, to appear, 2009.

[5] T. Bonald and L. Massoulié. Impact of fairness on internet performance. SIGMETRICS Perform. Eval. Rev., 29(1):82-91, 2001. ISSN 0163-5999. doi: http://doi.acm.org/10. $1145 / 384268.378438$.

[6] S. P. Boyd and L. Vandenberghe. Convex optimization. Cambridge University Press, 2004.

[7] E. Kalai and M. Smorodinsky. Other solutions to Nash's bargaining problem. Econometrica, 43:510-18, 1975.

[8] L. Kaplow and S. Shavell. Fairness versus welfare. Harvard University Press, 2002.

[9] F. P. Kelly, A. Maulloo, and D. Tan. Rate control for communication networks: Shadow prices, proportional fairness and stability. Journal of the Operational Research Society, 49:237-252, 1997.

[10] A. Kumar and J. Kleinberg. Fairness measures for resource allocation. In Proceedings of the 41st annual symposium on Foundations of Computer Science, pages 75-85, 2000.

[11] S. Kunniyur and R. Srikant. End-to-end congestion control schemes: utility functions, random losses and ecn marks. IEEE/ACM Trans. Netw., 11(5):689-702, 2003.

[12] T. Lan, D. Kao, M. Chiang, and A. Sabharwal. An axiomatic theory of fairness in network resource allocation. Working paper, 2009. http://arxiv.org/pdf/0906.0557.

[13] A. Mas-Colell, M. D. Whinston, and J. R. Green. Microeconomic Theory. Oxford University Press, 1995.

[14] L. Massoulié and J. Roberts. Bandwidth sharing: objectives and algorithms. IEEE/ACM Trans. Netw., 10(3):320-328, 2002.

[15] J. Mo and J. Walrand. Fair end-to-end window-based congestion control. IEEE/ACM Trans. Netw., 8(5):556-567, 2000. ISSN 1063-6692. doi: http://dx.doi.org/10.1109/90. 879343.

[16] J. Nash. The bargaining problem. Econometrica, 18:155-62, 1950. 
[17] R. Srikant. The mathematics of Internet congestion control. Birkhauser, 2004.

[18] A. Tang, J. Wang, and S. H. Low. Counter-intuitive throughput behaviors in networks under end-to-end control. IEEE/ACM Trans. Netw., 14(2):355-368, March 2006.

[19] H. Peyton Young. Equity: In Theory and Practice. Princeton University Press, 1995. 